\title{
Evolution of plasmonic response in growing silver thin films with pre-percolation non-local conduction and emittance drop
}

\author{
A A Earp and G B Smith ${ }^{1}$ \\ Department of Physics and Advanced Materials and INT, University of \\ Technology, Sydney, PO Box 123, Broadway NSW 2007 Australia \\ E-mail: g.smith@uts.edu.au
}

\begin{abstract}
The optical response of growing silver thin films undergoes a transition dominated by three distinct plasmonic modes, two localized and one delocalized. Their admix as a function of added mass is analysed. The onset of the delocalized or Drude mode occurs before the sharp electrical percolation transition so optically the full insulator-metal transition is broadened. A scaling explanation supported by images shows Ag islands only have to link up over 200 to $300 \mathrm{~nm}$ to yield partial delocalization. The localized modes are (i) from silver nano-islands and (ii) a transitional anomalous mode, peaking near the dc critical percolation point, from islands surrounded by network. Growing silver within a multilayer oxide stack is compared with that on glass. The transition in thermal emittance matches that in the delocalized mode. Its broadening enables practical tuning of intermediate emittance by varying mass.
\end{abstract}




\section{Introduction}

Vacuum deposited silver and gold thin films initially grow as nano-islands. With further deposition partial clustering occurs followed by formation of extended conducting networks. Finally a porous phase persists until all nanopores vanish [1-5]. The dc electrical response across this growth domain is well characterised by classical percolation/phase change theory $[3,6,7]$ with conductivity under-going a sharp phase change which varies as $\left(f-f_{c}\right)^{ \pm \alpha_{ \pm}}$with $\mathrm{f}$ metal volume fraction. The critical co-efficients $\alpha_{ \pm}$are not independent of the critical concentration $f_{c}$ $[6,7]$ but at large enough scales both quantities are insensitive to local variations in nanostructure. They instead involve averages which can be expressed in terms of a dimensionality or connectivity $d$ of the network [8]. Parameters $d, \alpha$, and $f_{c}$ are thus not independent and vary with average connectivity which is hyper-dimensional when $d>3$ [6]. Growing thin films involving islands linking up are usually allocated $d=2$ for silver or $d=3$ for gold. Since the voids in these growing films extend vertically across the layer they would be considered optically to be a 2-dimensional structure, so observing $d=3$ from criticality exemplifies the distinct role of connectivity. It clearly differs in growing Ag and Au. This in turn is linked to differences in atomic diffusivity [6].

New insights into the transitional changes in optical behaviour across this whole growth domain, and how and why they differ from the electrical transition, is our focus in this paper. These shifts in optical response as a function of mass deposited cannot be described in usual macroscopic phase transition terms, especially near percolation, since more nanostructure details apart from average connectivity come into play. However since reflectance and transmittance data is obtained in the "far field" some averaging over fine structural or "near field" detail remains. Recent work [9] has shown that widely varying near field intensities in such films are correlated only over around $100 \mathrm{~nm}$ distances so detail across a wavelength are expected to be washed out in the far field averaging process. Another way of thinking about this is embodied in the success of effective medium approximations to optical response [10]. In such models local nanoscale fields induced under illumination are spatially averaged resulting in homogenised values of induced polarisation and an effective permittivity. A few wavelengths is a reasonable guide to the scale of interest for averaging. Thus if dc percolative phase transitions are probed over similar finite scales, instead of typical macroscales, partial conduction onsets are expected at $\mathrm{f}$ values in advance of the infinite scale critical concentration. This has in fact been observed and modelled in one study for electrode spacings from $300 \mathrm{~nm}$ to $3200 \mathrm{~nm}$ [11]. As the spacing between 
electrodes shrinks towards the size of typical nanofeatures, conduction onsets occurred at successively lower concentrations while the transition width broadened considerably. Conductors are also plasmonic when their dielectric constant is negative which occurs in the optical or IR domain and depends on carrier concentration. However plasmonic response is quite distinct spectrally depending on whether charge carriers are localized or de-localized. It is the delocalized component that traditionally links to dc conduction and we will use as a marker of percolation response. An intriguing aspect we show is the very small number of islands that have to link up before some delocalization is observed while the overall transition is broadened just like that of dc response when electrode spacings are nanoscale.

Our study covers the full visible-near IR spectrum (400 nm to $2500 \mathrm{~nm}$ ) and also the thermal emittance. The emittance percolation transition is technically important and interesting. We shall show that the onset of a fall in thermal emittance also occurs at $f$ values well below $f_{c}$, while its overall transition is also broadened, a feature which enables precise emittance engineering.

An optical modelling procedure that defines response at all stages of growth has been developed and is described in the next section. For silver it is found that an admix of three distinct types of plasmonic responses are adequate, two of which are localized and one is delocalized. In addition a near constant background permittivity from high energy inter-band Ag transitions is needed. Different relative weights of each plasmonic component as growth proceeds shows clearly the dominant structure-optical impacts near percolation and for the delocalized component transition broadening relative to the dc case. A procedure similar to ours has been applied to thin gold films recently [5]. The complexity of inter-band terms in gold, plus using bare non epitaxial, rather than buried films and having less samples near percolation meant some key aspects that are clear in silver were not obvious. They did however see but couldn't explain the anomalous transitional plasmonic peak which we have previously structurally explained $[15,17]$ and whose novel nature had meant many previous attempts at effective medium modelling failed near percolation. A related procedure based on a varying spectral density representation has been applied recently to observed percolation in $\mathrm{Co}-\mathrm{Al}_{2} \mathrm{O}_{3}$ nano-composites as Co particle content varies [12]. This generalized mathematical approach has a qualitatively similar low mass evolution to our direct physical method in terms of admix of spectral components but in contrast runs into quantitative difficulties near and beyond percolation. A detailed future comparison of the two methods on the same samples would thus be worthwhile. Differences in ability to handle percolation may be linked to the different topologies in growing noble films and arrays of metal nanoparticles, to 
differences in the way changes in key parameters such as loss and metal indices are treated, or to the different mathematical manner in which the two approaches handle broadening due to multiple overlapping resonances.

\section{Plasmonic model for evolution of Ag thin film optical response}

The asymptotic structures either side of percolation are island silver at low mass and nanoporous interconnected silver then dense silver at sufficiently high mass. These have been adequately modelled in the past with single plasmonic oscillators. The island oscillator is that for surface plasmon resonance (SPR) on nanoparticles [10, 13]. Sufficiently beyond percolation a delocalized plasmon oscillator dominates response. It has Drude like character with initially an effective plasma frequency $\omega_{\mathrm{P}}^{*}$ linked to silver density [14], and finally once porosity vanishes that for fully dense silver. The particle oscillator could involve broadening from more than one overlapping oscillator (e.g. from shape variations), but almost always manifests as a single absorption peak in optical data. Near percolation two questions arise (i) what is the admix of these two asymptotic resonances? (ii) Can significant additional resonances be observed in the far field and if so how many? If additional resonances arise their nanostructural origins are also of interest.

Experimentally only one distinct, additional absorption resonance has been clearly identified and only appears near percolation $[5,15]$. It is hard to distinguish when the insulator phase is just voids being from a quite dilute component, which helps explain why it has only been recently identified. A qualitatively similar but much stronger resonance arises in nanoporous metal made by etching [16]. It becomes obvious in these films, despite its low concentration, when the voids are filled with insulator. This happens when oxide/noble metal/oxide multi-layers are made, as used in energy efficient glazing, but with metal layer growth truncated before densifying. Such samples are used in this study. This intermediate resonance is also plasmonic in origin and can be understood in terms of a new type of "effective resonant unit" in which an isolated metal particle is separated from surrounding conductor by a thin insulator shell $[15,17]$. This unit is the Babinet's counterpart structure of another well-known SPR system namely an insulator nanoparticle, surrounded by a thin metal shell embedded within an insulator [18]. The Babinet's SPR resonance is much stronger than its better known insulator core-metal shell counterpart. In 
the problem here the "conducting matrix" is not dense but a small scale nanoporous network. At any mass deposited the effective oscillator model of equation (1) for observed relative permittivity $\varepsilon(\omega, \mathrm{t})$ at frequency $\omega$ (in $\mathrm{eV}$ ) and mass thickness t of silver, is able to both fit all of our data and provide significant new physical insights into the optical transition.

$$
\varepsilon(\omega, t)=\varepsilon_{\infty}(t)+\sum_{i=1}^{3} \frac{A_{i}(t)}{\omega_{i}^{2}-\omega^{2}-i \gamma_{i}(t) \omega}
$$

$\mathrm{A}_{\mathrm{i}}(\mathrm{t})$ in units $(\mathrm{eV})^{2}$ is the spectral weight of plasmon resonance $\mathrm{i}$ at mass thickness $t . \omega_{i}$ is the resonant frequency with $\omega_{\mathrm{i}}=0$ when $\mathrm{i}=1$ which is the de-localized or Drude like response. $\gamma_{1}(\mathrm{t})$ is then the usual conduction electron relaxation rate (in $\mathrm{eV}$ ) and can vary with t due to changes in surface collision contributions to carrier loss as nanostructure varies. We will use labels $\mathrm{i}=3$ for the island silver SPR resonance and $\mathrm{i}=2$ for the anomalous metal in conductor SPR found near percolation. $\gamma_{i}(t)$ for $i=2$ and 3 is a resonant broadening parameter. It will have a contribution from carrier relaxation, and in these complex structures its value will also be affected by overlapping of neighbouring resonances. Overlap of isolated island SPR's can result from shape variations and for the Ag core-insulator shell in conductor SPR's from variations in core shape and almost certainly from variations in effective shell thickness relative to core radius. The latter SPR's resonant wavelengths are quite sensitive to this geometric ratio [15,17]. It is interesting however that in the data that follows $\omega_{2}$ is found to be almost constant at $0.989 \mathrm{eV}(1.25 \mu \mathrm{m})$ implying that as $\mathrm{t}$ varies the distribution of core radius to effective shell thickness ratio does not change.

Models of the observed spectral permittivity found using equation (1) with an effective medium model are in principle possible. Our results require that four structural components be included; isolated particles, linked metal, insulator, and the metal core-insulator shell in network entity. A reduction to a three-component model may be feasible if the network plus core-shell is first considered as one material, in a two stage effective medium approach. Volume fractions $f$ which vary with t should be found from high resolution images. Past attempts to model the percolation transition in growing metal films as an effective medium approximation (EMA) usually relied on a two phase Bruggeman $(\mathrm{Br})$ model $[6,7,19]$ because it allowed for formation of a touching network of nanoparticles to form at a critical concentration, for example at $f_{c}=0.33$ for gold 
spheres. A phenomenological modification to the Br model has been introduced [20] in which component dc conductivity's dependence on $\left(f-f_{c}\right)$ is exponential with critical coefficients. This approach does reproduce the observed behaviour of macroscopic $\sigma(f)$ as $f$ approaches $f_{c}$ namely as $\left(f-f_{c}\right)^{ \pm \alpha_{ \pm}}$. However as we shall see the optical transition is not this sharp (due to the small scale of the applied field), so such a phenomenology needs changing. The optical transition does however have its halfway point close to the dc electrical values of $t_{c}$ and $f_{c}$.

\section{Experimental analysis}

\subsection{Sample Preparation}

Two series of Ag thin films were deposited for this study using DC magnetron sputtering, with mass thicknesses based on pre-calibrated deposition rates ranging from $2.0 \mathrm{~nm}$ to $12.0 \mathrm{~nm}$. Series A was deposited on glass substrates with dimensions 25mm x $75 \mathrm{~mm}$ x $1 \mathrm{~mm}$, using a CS 12/14 high vacuum coating unit (Dynavac) under pressures of approximately $6.7 \times 10^{-4} \mathrm{~Pa}$. Sputtering was carried out under Argon pressures of 0.37 Pa and a DC current of 0.05 A. The Ag deposition rate was calibrated three times using films approximately $50 \mathrm{~nm}$ thick and thickness was fitted in WVASE32 spectroscopic software (Woollam Co., Inc.) using measured transmittance spectra. The Ag mass thicknesses calculated from the deposition rate were found to be accurate to within $1 \%$.

For series B, the Ag films were deposited in the middle of a multilayer thin film stack, with thin film $\mathrm{ZnO}$ beneath the Ag layer, and aluminium-doped zinc oxide (ZAO) directly above. These samples were produced using an industrial magnetron sputtering unit on $3.8 \mathrm{~mm}$ thick float glass substrate as used in normal windows, and then cut into $100 \mathrm{~mm}$ x $100 \mathrm{~mm}$ samples. The Ag layer thickness was again varied in these samples from $2.0 \mathrm{~nm}$ to $12.0 \mathrm{~nm}$. All layers in the multilayer stack were deposited at consistent rates and the individual oxide layers were also independently optically characterised after stand-alone depositions. Most of the experimental data presented and analysed in detail in this paper was derived from the series B multilayer films since as noted above the intermediate resonance near percolation stands out more when the nano-voids in silver are filled. This is also the case most relevant to use in buildings.

\subsection{Structural Characterisation}

The surface structure for the series B films was measured with a Zeiss Supra 55VP 'Field Emission' Scanning Electron Microscope (SEM). The SEM images presented in Figure 1 at four different mass thicknesses have a magnification of 200,000 times, and were obtained under high 
vacuum of $3.2 \times 10^{-8} \mathrm{~Pa}$, with a $20 \mathrm{kV}$ source voltage and an extractor current of $168 \mu \mathrm{A}$. To enhance image contrast, a layer of copper conductive tape was used to provide an electrical connection between the top surface of the film and the detector.
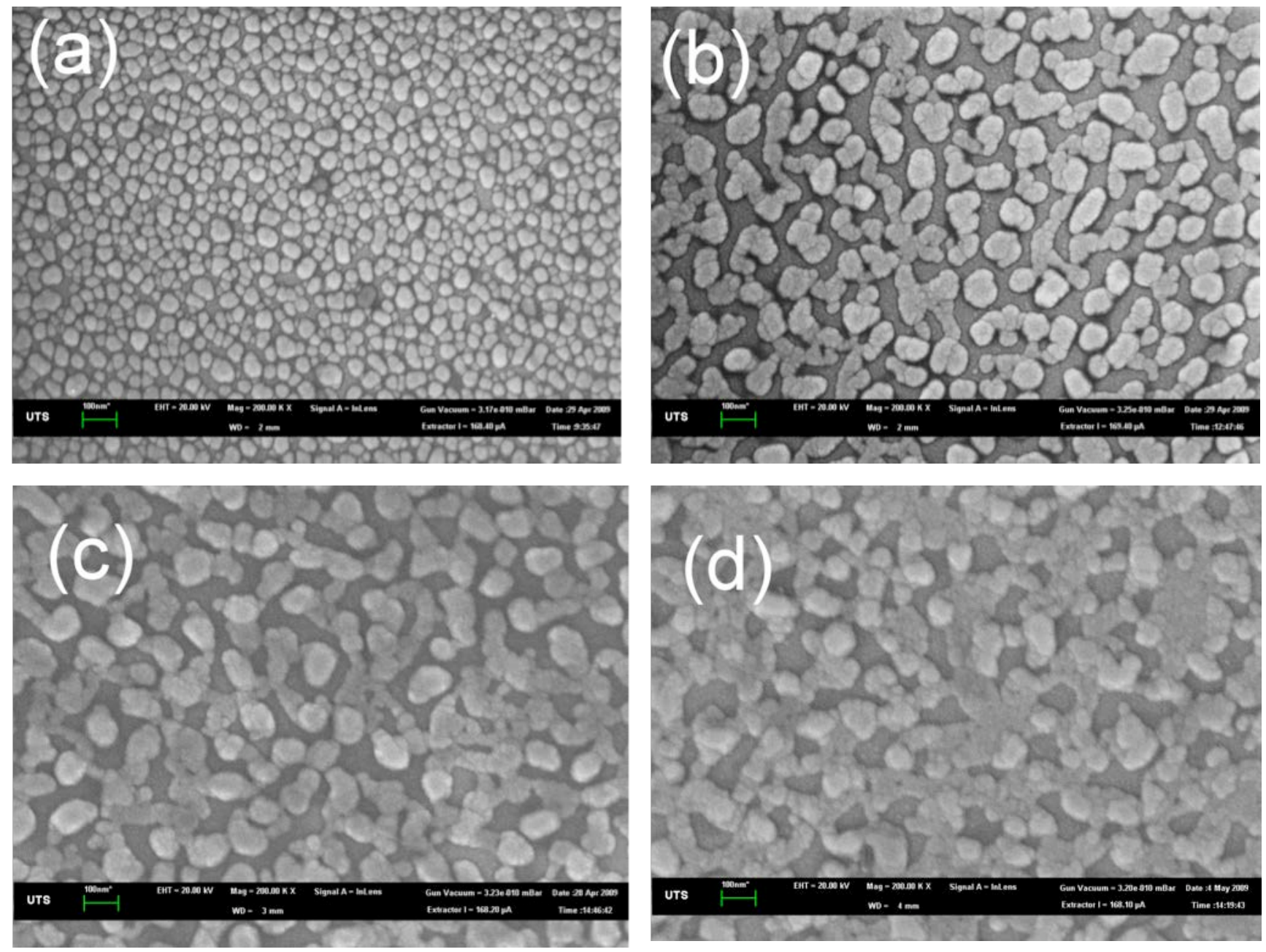

Figure 1. High resolution images of various mass thicknesses of thin Ag films on $\mathrm{ZnO}$ within a multi-layer stack. (a) $2.3 \mathrm{~nm}$ (b) $4.3 \mathrm{~nm}$ (c) $4.7 \mathrm{~nm}$ (d) $5.4 \mathrm{~nm}$.

\subsection{Spectral Measurements}

Transmittance and reflectance spectra were measured over the range $300-2500 \mathrm{~nm}$, spanning the ultraviolet (UV), visible and near-infrared (NIR) wavelength regions as needed for solar control 
glazing, using a Varian Cary 5E UV/Vis/NIR spectrometer. Results appear in figure 2 for a complete range of multi-layer stacks in terms of silver film mass thickness, with the remainder of the stack fixed and $\mathrm{ZnO}$ the thin film underlying the Ag layer.
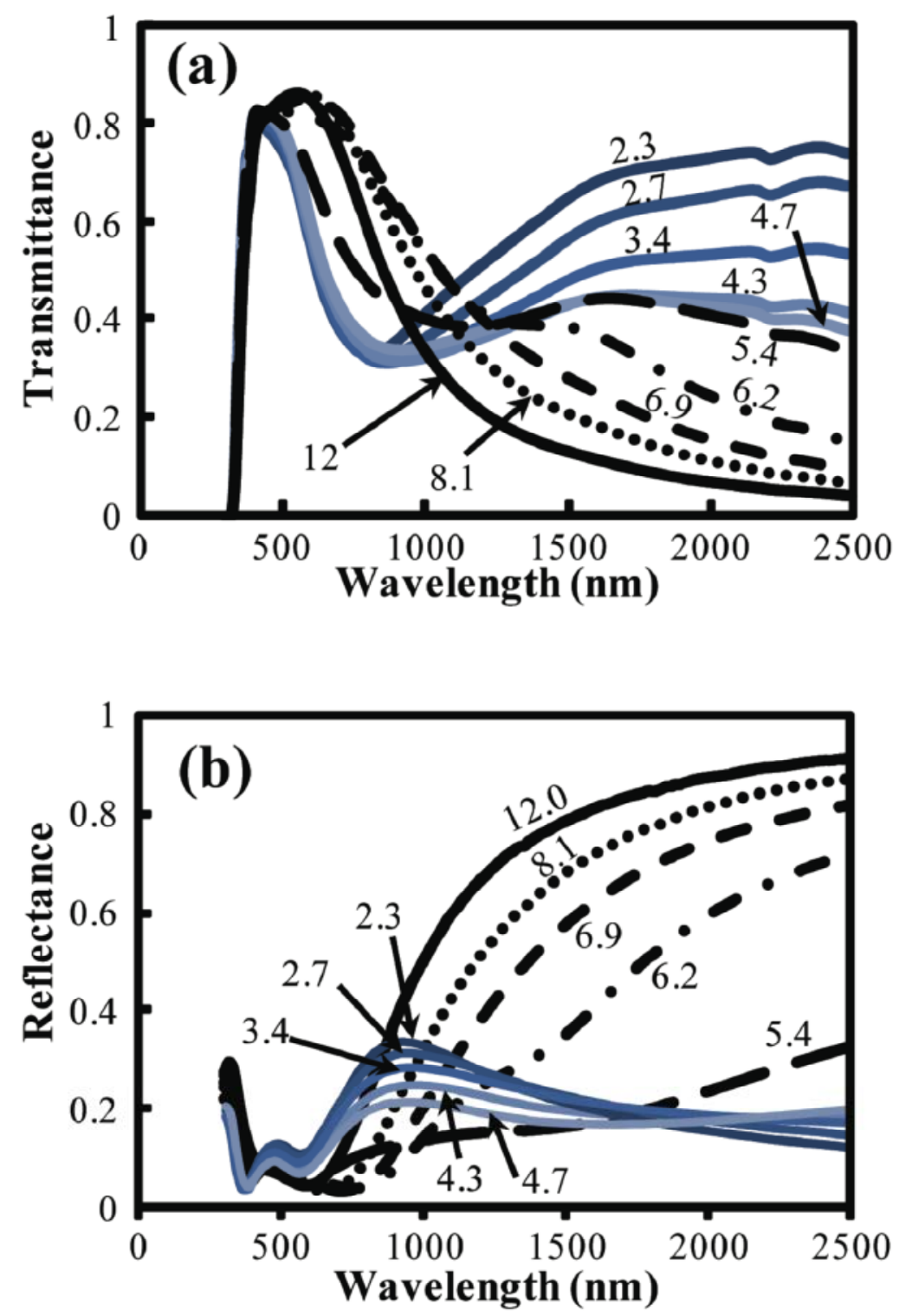

Figure 2 Measurements on composite thin Ag films on $\mathrm{ZnO}$ within a multi-layer stack for a wide range of mass thicknesses in nm, shown numerically as plot labels. (a) spectral transmittance (b) spectral reflectance.

For these series B window samples the float glass was $3.8 \mathrm{~mm}$ thick and had an Fe absorption band in the region $550-1500 \mathrm{~nm}$, so the transmittance and reflectance of the float glass substrate 
were measured in order to isolate the absorption due purely to the film stack and finally the silver layer itself. The spectral absorption $A_{g}$ of this glass substrate at wavelength $\lambda$ was calculated using:

$$
A_{g}(\lambda)=1.0-T_{g}(\lambda)-R_{g}(\lambda)
$$

with $\mathrm{T}_{\mathrm{g}}=$ bare glass transmittance and $\mathrm{R}_{\mathrm{g}}=$ bare glass reflectance. Similarly, the total spectral absorptance for each multilayer film sample was calculated using equation (2) with subsript " $g$ " replaced by subscript "tot" so $T_{\text {tot }}(\lambda)$ is the total transmittance of the multilayer film stack on float glass and $\mathrm{R}_{\mathrm{tot}}(\lambda)$ is the reflectance of the sample with its film side facing towards the light source. To exclude transmission losses in the glass substrate, the transmittance of the stack into the glass substrate $\mathrm{T}_{\mathrm{fg}}$ was first calculated by dividing the total transmittance by that of the substrate $\left(\mathrm{T}_{\text {tot }}(\lambda) / \mathrm{T}_{\mathrm{g}}(\lambda)\right)$. The subscript 'fg' thus includes the impact of the Ag film plus surrounding oxides, since the light travels first through the film stack then the glass. For the measurement of substrate absorption $A_{g}(\lambda)$ in Eq. (1) above, there was no film on the substrate. Absorptance then impacts on light transmitted after front air/glass interface reflectance $r(\lambda)=$ $\left[(n-1)^{2}+k^{2}\right] /\left[(n+1)^{2}+k^{2}\right]$. These $\mathrm{n}, \mathrm{k}$ optical constants, which include the effects of the iron absorption, were derived in WVASE32 software from measurements of $T_{g}(\lambda)$ and $R_{g}(\lambda)$ for the glass substrate.

The absorption due to the multilayer film stack $A_{\text {film }}$ is then calculated by subtracting the absorptance due to the glass when under the film stack (labeled here $\mathrm{A}_{\text {guf }}$ ) from the total measured absorptance $A_{\text {tot }}$. $A_{\text {guf }}$ is different to $A_{g}$ by a factor that arises because the contribution to absorption of the glass substrate underneath each film must allow for the fact that light is absorbed by the film before it reaches the glass. The result is

$$
A_{\text {guf }}(\lambda)=\frac{T_{f g}(\lambda)}{T_{0}(\lambda)} A_{g}(\lambda)=\frac{T_{f g}(\lambda)}{1-r(\lambda)} A_{g}(\lambda)
$$

Where $A_{\text {guf }}(\lambda)$ is the applicable absorptance of the glass when underneath the film. For the series A samples with just Ag on glass, the substrate was $1 \mathrm{~mm}$ thick and had a negligible absorption profile over the wavelength range of interest, so the absorption of the films was calculated by subtracting the absorption of the measured glass substrate A from the total absorption. The results of this analysis are experimental spectral absorptance $A_{\text {film }}$ for the stack itself, which occurs almost entirely within the silver layer at visible and NIR wavelengths and is plotted in figure 3. 

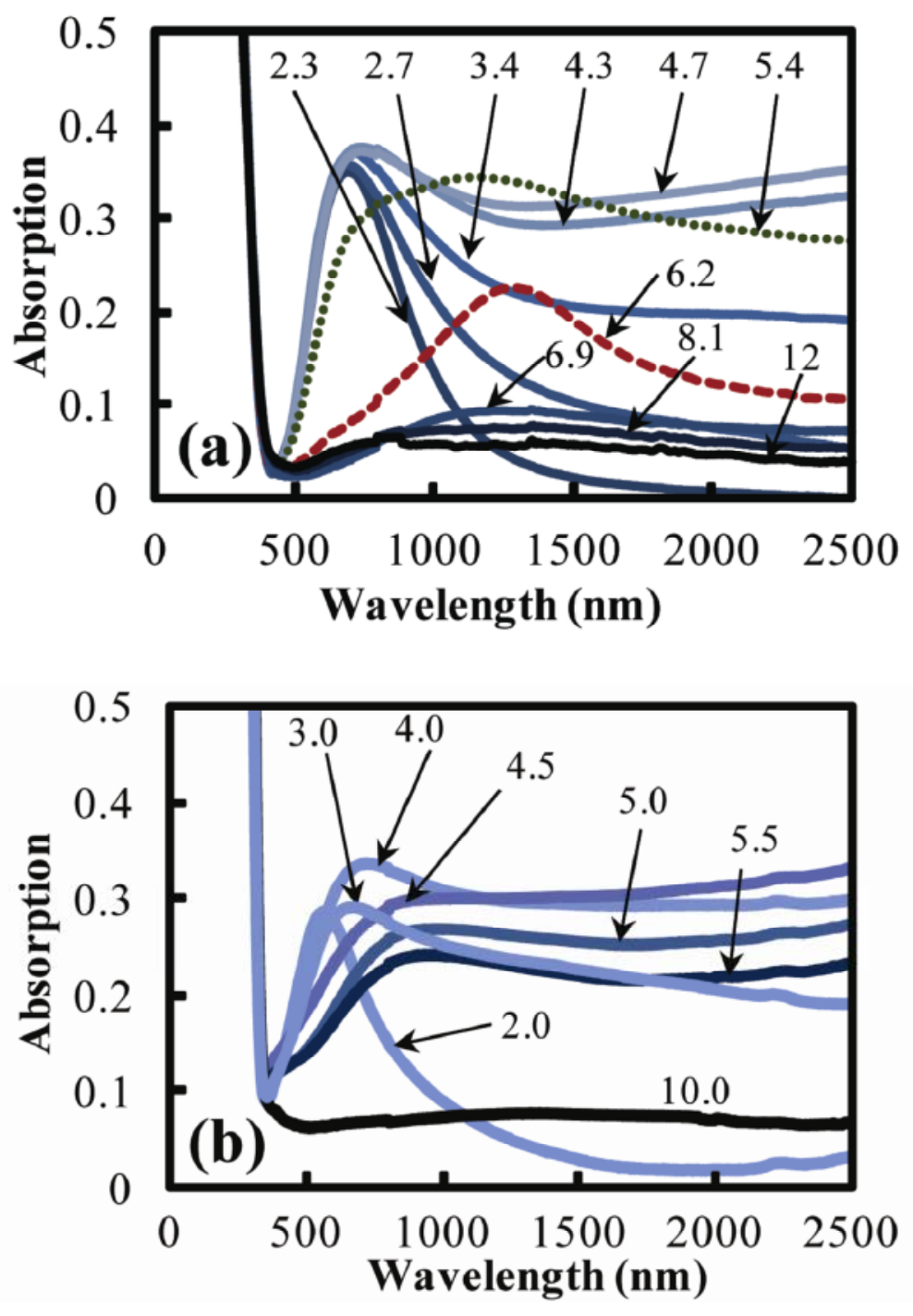

Figure 3. Measured absorptance in growing Ag thin films at increasing mass thicknesses labeled in nm (a) voids filled with insulator inside multilayer stack (b) on glass with film silver plus voids

\subsection{Modelling Optical Constants}

The optical constants of the thin films were determined with WVASE32 spectroscopic and ellipsometric software (by J.A. Woollam Co., Inc.), using equation (1). Transmittance and reflectance spectra were generated from the modeled optical constants, and compared with the experimentally measured spectra for each sample. Goodness of fit was determined by calculating the mean squared error (MSE), as follows. 


$$
M S E=\frac{1}{2 N-M} \chi^{2}
$$

Where $\mathrm{N}$ is the number of data points, $\mathrm{M}$ is the number of variable parameters in the model, and $\chi^{2}$ is the sum (for all spectral data points) of the squares of the ratio of the difference between experiment and model values to the experimental value.

The optical constants of each layer in the multilayer stack except for silver, were modeled using experimental spectral measurements of the oxide layers by themselves. Individual thicknesses also were fitted and used to calibrate deposition rates for these oxides. Models of the multilayer films were then constructed using these optical constants and initially expected film thicknesses for the non-silver layers based on their calibrated deposition rates. Oscillator strengths and film thicknesses of the silver layer and finally if necessary minor adjustments to thickness of the other layers, were adjusted systematically until the optimal match between modeled and experimental data was achieved for the multilayer sample with the thickest Ag layer $(12 \mathrm{~nm})$. This void free silver layer was a good test as it was expected to have close to bulk silver constants apart from a slight increase in Drude relaxation rate, which is itself low for a thin film due to the impacts of the oxide layer below on grain structure. The resulting stack structure and optical constants of all layers except silver were then carried through to the remainder of the multilayer film samples. Experimental measurements of transmittance, film side reflectance and glass side reflectance were fitted to provide the final model parameters for each embedded silver layer. The final models gave a fit to the measured data, with mean squared error (MSE) values between 0.93 and 1.72 over the wavelength range $300-2500 \mathrm{~nm}$. This is regarded as a good fit over such a wide range. Results for the real part of dielectric constant $\varepsilon_{1}$ for the composite Ag containing films within the stack are plotted in figure 4, which demonstrates clearly the transition as t increases from homogenized response which is insulating to homogenized response which is plasmonic at longer wavelengths.

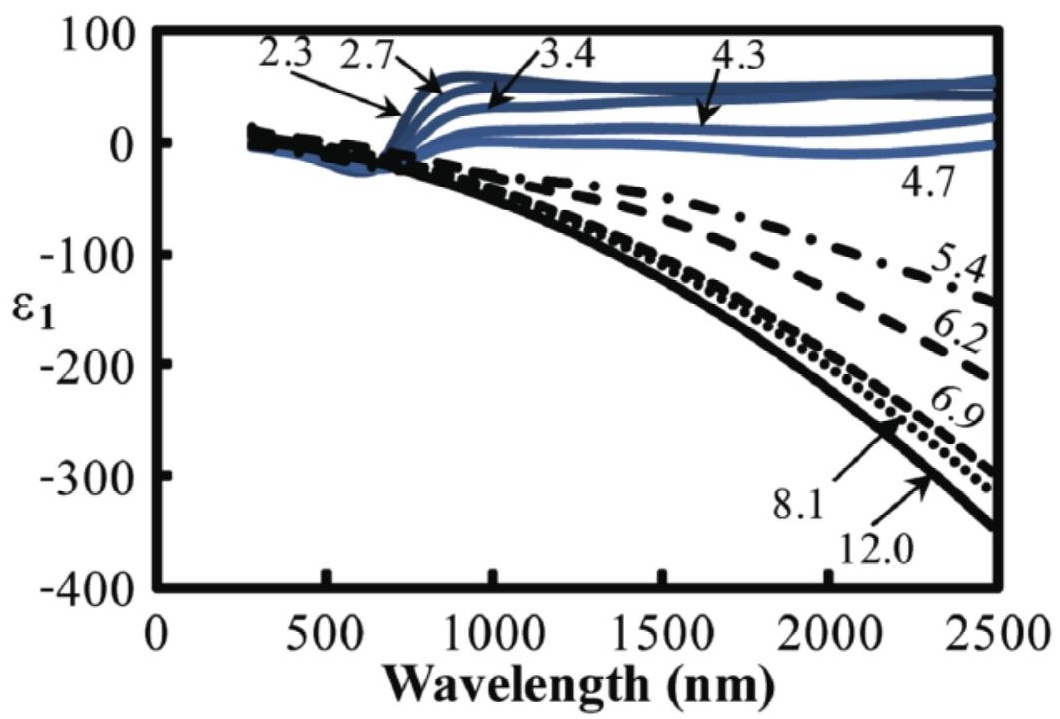


Figure 4. Real part of the dielectric constant of the composite silver films within the stack as mass thickness increases.

The three primary oscillators are all plasmonic in nature as noted above and were clearly identified for the Ag layers within the multilayer films; (i) Drude type oscillator, at $0 \mathrm{eV}$ due to delocalized electrons (ii) local oscillator at $0.989 \mathrm{eV}$ due to isolated core-shell systems inside a small network (iii) oscillator at $1.60 \mathrm{eV}$ due to groups of isolated Ag nanoparticles. The $1.6 \mathrm{eV}$ oscillator was only observed up to Ag thicknesses of $5.4 \mathrm{~nm}$, as the particle groups were then largely replaced by conducting networks. The complete mathematical fit to the data also involved small adjustments to the constant $\varepsilon(\infty, \mathrm{t})$. Such adjustments as silver mass thickness $t$ increases were expected and reflect the increasing contribution of high energy silver inter-band terms when more silver is present.

\subsection{Thermal and Electrical Characterisation}

The thermal emittance of the films was measured using a Windbourn rotating dual cavity emissometer in which the cavity irradiating the sample is heated to about $100^{\circ} \mathrm{C}$. The proportion of this radiation reflected to the compact thermopile detector is sampled and fed to a lock-in amplifier. A copper sample with emittance of $e=0.04$ was used as a reference standard. Emittance was measured six times for each sample, so the maximum uncertainty in the mean value of $e$ is estimated to be \pm 0.005 .

For each film, the electrical resistance was measured with a digital multimeter (Yokogawa 7562) over the range $1 \Omega$ to $10 \mathrm{G} \Omega$, by painting $3 \mathrm{~mm}$ x $6 \mathrm{~mm}$ Ag electrodes onto the top surface of the film, separated by a distance of $3 \mathrm{~mm}$. As the 2-3 nm thick Ag layers consist of isolated Ag nanoparticles, these films have electrical resistances of the order of $10 \mathrm{G} \Omega$, while the 8-12 nm thick layers are electrically conducting, with resistances of the order of 5-10 ohms. Each sample 
was measured between 3 and 6 times, and the mean resistance values were calculated, providing relative uncertainties of less than $5 \%$.

\section{Analysis of optical, thermal and electrical percolation}

In this section the evolution of optical response as shown in figures 2, 3, 4 with growth in thin silver films, is correlated with changes in plasmonic response, which in turn is linked to changes in nanostructure. Optical response near percolation as mass thickness changes is also compared with changes in dc response. Some important differences are clearly apparent and are discussed. Since thermal emittance $e$ is an important technological aspect of silver thin films, especially when embedded within multi-layers, we also examine in this section its rate of change near percolation in comparison to shorter wavelength optical properties and to dc response.

As noted in the introduction the onset of delocalized properties depends on how far the network extends in relation to the scale of the experimental probe of interest. Classical theory requires an infinite range and results in an infinitely sharp transition. Most dc measurements are close approximations to this limit but we now show that optical and thermal radiation fields involve broadened transitions.

At all scales the onset of percolation does not mean that all conducting material belongs to the network. dc response is typically however dominated by the network part once it forms whereas optical and thermal response still involves significant contributions from non-network conducting material as we now show. Figure 4 is our result for optical fitting of our full range of silver films in a multi-layer stack using equation (1). It shows the evolution of the spectral weight or oscillator strength of the three generic plasmonic components; groups of isolated silver nanoislands (A3), single silver islands surrounded by but separate from the conducting network (A2), and the small scale network which is represented by a Drude like response. This Drude strength, and hence effective plasma frequency of the network, does not appear to reach that expected for dense silver in these films until a mass thickness near $12 \mathrm{~nm}$. 


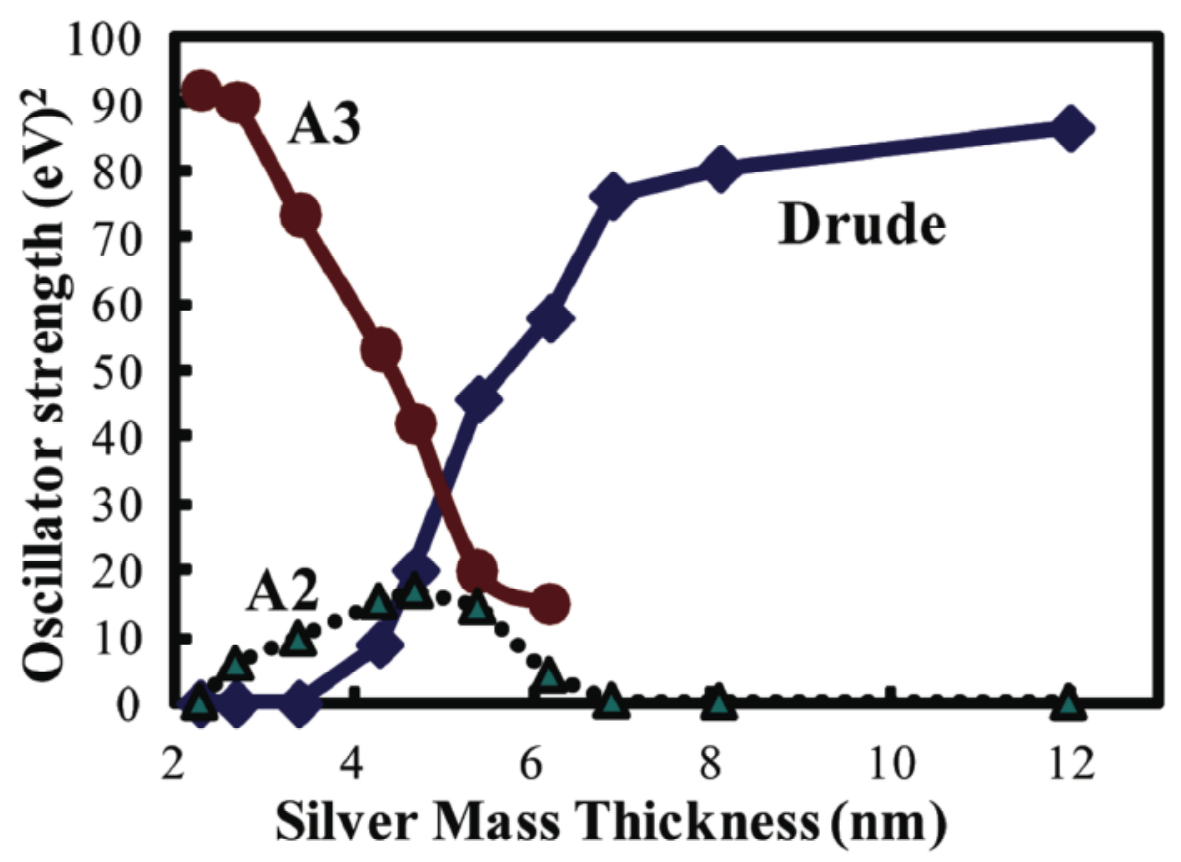

Figure 5. Admix of the three dominant plasmonic components at various stages of growth in silver thin films. A2, A3 are localized resonators while the Drude term is the delocalized component.

It is also of some interest that the delocalized response defined by having $\omega_{\mathrm{i}}=0$, and oscillator A2 response, show up well before the percolation point near $5 \mathrm{~nm}$ mass thickness, while some regions of isolated islands persist beyond percolation. It is also interesting that oscillator A2 shows up when the Drude contribution is still quite weak. It peaks as expected from its nature, close to the percolation cross-over point. Defining the percolation point is not as straightforward optically as it is for the sharp dc electrical response. Our analysis using optical criteria indicates it occurs at a slightly higher mass than electrical percolation despite its earlier partial onset. A2's weight when the Drude response is still weak may come partly from its intrinsic strength per particle which is much stronger than A3 type resonances (as detailed formally in reference [17]) but its early onset does require further analysis. The images (b) and (c) in figure 1 when viewed from the perspective of this data indicate that clustering of silver over ranges of a few hundred $\mathrm{nm}$, that is around a wavelength in scale, is sufficient to create some delocalized or Drude type response as well as A2 type response in the far field. This also touches upon a fundamental issue for nanostructured plasmonic materials, namely at what length scales do surface charge effects 
and hence localized surface plasmons, drop out optically in favor of delocalized plasmonic response.

Electrical and optical percolation are now compared. Two measures of percolation involving a range of wavelengths are used here (i) the Drude weight from spectral analysis (ii) measured thermal emittance. As seen in figure 6 both of these quantities are closely linked and evolve in almost identical fashion as mass deposited increases with the mid-point in their transition each near $5 \mathrm{~nm}$. We take this mid-point as a practical definition of the optical transition. Both clearly involve the Drude contribution to spectral response since as any material changes from an insulator to a conductor its e value must undergo a large change from a high value around 0.8 0.9 to a low value which for noble metals is under 0.1 . It can be seen that emittance starts to fall at quite low silver mass, which again points to some delocalized conduction at these frequencies occurring over quite small scales. The correlation of emittance change to resistance change with mass added is particularly interesting as seen in figure 7 .

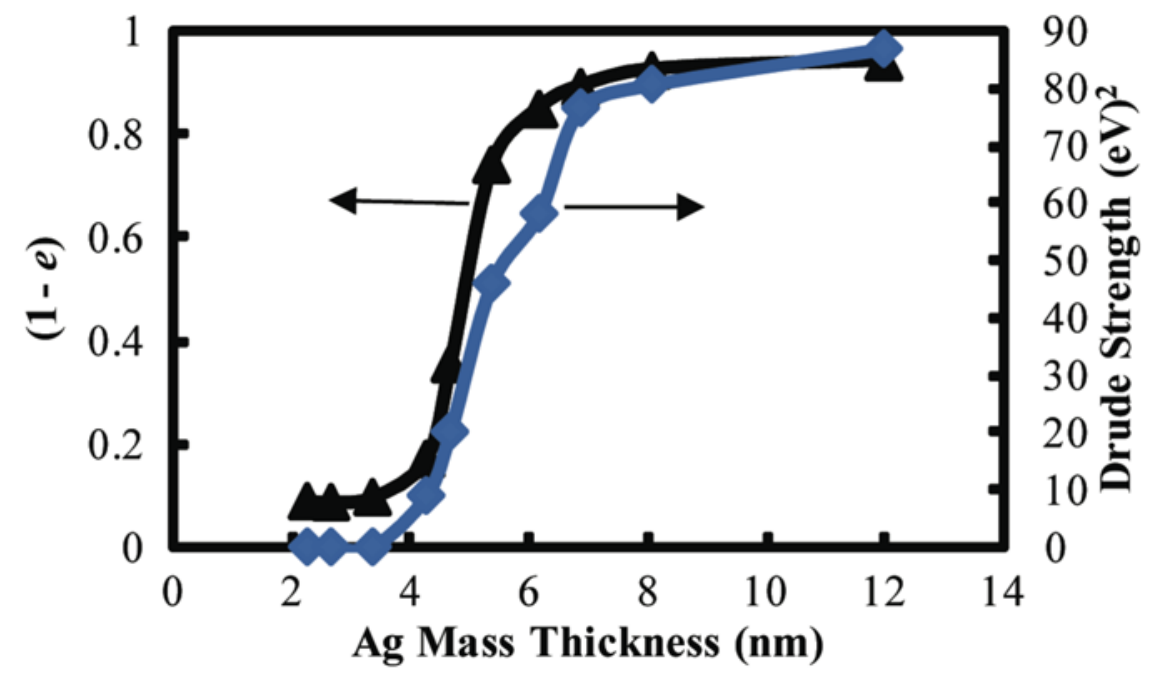

Figure 6. A comparison of thermal emittance e and Drude spectral weight $\mathrm{A}_{1}$ as a function of $\mathrm{Ag}$ mass thickness. $(1-\mathrm{e})$ is plotted to highlight their close correlation. 


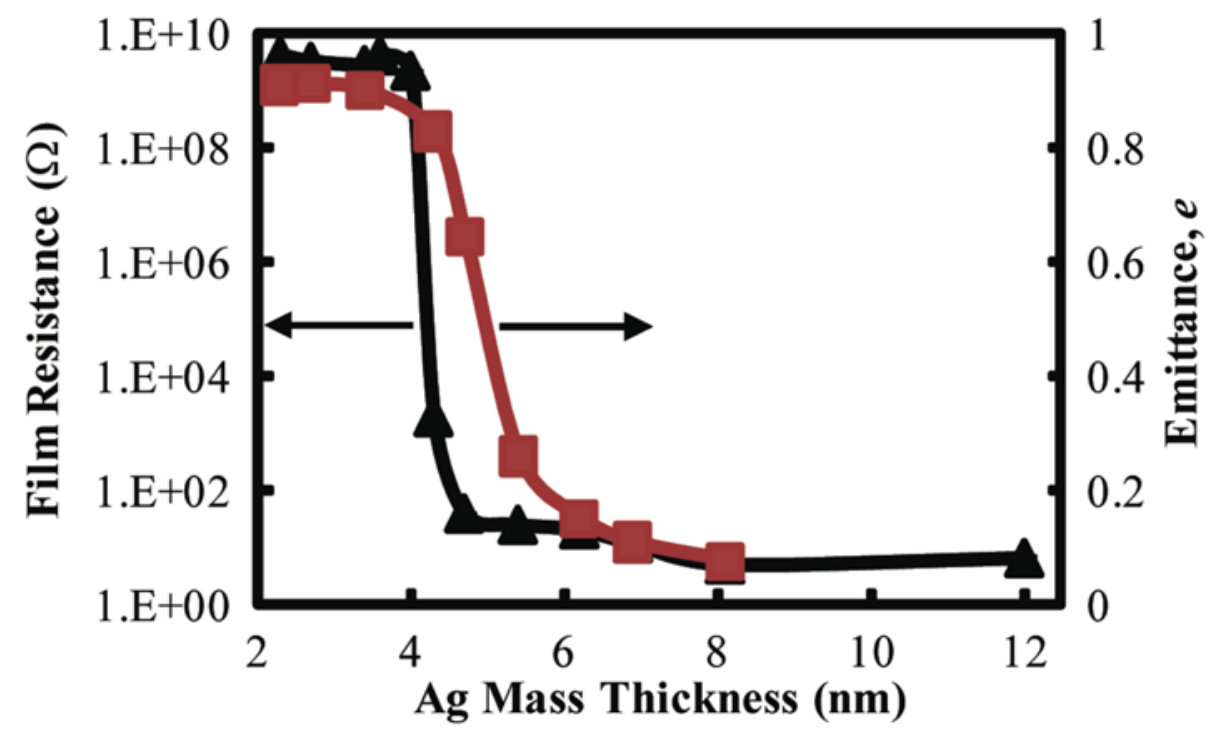

Figure 7. A comparison of changes in electrical resistance and thermal emittance with mass of Ag deposited. The percolation point appears to be lower for dc resistance but the onset of evidence for extended conduction is lower in emittance.

In figure 7 it appears that the sharper dc transition occurs at lower mass deposited of about $4.2 \mathrm{~nm}$ compared to that for both emittance and the Drude component of about $5 \mathrm{~nm}$. However the onset of a fall in emittance occurs at a much lower added mass than the onset of dc response as expected if it involves conduction over a much smaller scale. It is also worth noting from figs 6 and 7 that these granular metal films inside a multilayer provide a precise engineering means of controlling thermal emittance over a wide range. A broader transition than that found in resistivity actually facilitates practical emittance control whereas a very sharp transition as in dc data would mean achieving controlled e values in the range 0.8 to 0.2 would be difficult, if not impossible, to reproduce.

\section{Conclusion}

Three dominant plasmonic modes contribute to optical response in growing silver thin films, two of which are observed via single resonant peaks associated with localized surface plasmons. The evolution of their admix, as measured by spectral weights, has some surprising features. The onset of delocalized or Drude like response appears at very low added mass, long before evidence of dc electrical response. The reduced Drude spectral weight, which occurs in all nanoporous layers depends on the porosity. Of the other two dominant plasmonic contributions that from 
metal islands is expected while the other involves a relatively new concept. It arises from the Babinet's counterpart to insulator core-metal shell nanoparticles in insulator, namely a metal coreinsulator shell entity surrounded by delocalized or Drude component.

These findings support finite scaling models of percolative phase transitions [11]. Partial onsets of the delocalized phase is seen optically long before the classical "infinite" network forms as the probing fields have nanoscale or micron scale sizes. In addition the complete transition is broadened. Transitional novel phases, which vanish at high mass, also come into play optically at these scales. This broadening has useful practical implications for example granular metal films can be used to precisely engineer intermediate values of thermal emittance.

\section{References}

[1] Smith G B, Niklasson G A, Svensson J S E M and Granqvist C G 1986 J. Appl. Phys. 59571

[2] Gadenne P, Beghdadi A and Lafait J 1988 Optics Commun. 6517

[3] Hovel M, Gompf B and Dressel M, 2010 Phys. Rev. B 81035402

[4] Seal K, Nelson M A and Charles Z 2002 J. Modern Optics 492423

[5] Byon E, Oates W H and Anders A 2003 Appl. Phys. Lett. 821634

[6] Smith G B, Maaroof A I and Cortie M B 2008 Phys. Rev. B 78165418

[7] Clerc J P, Giraud G, Laugier J M and Luck J M 1990 Advances in Physics 39191

[8] Chaikin P M and Lubensky T C Principles of Condensed Matter Physics (Cambridge University Press, Cambridge, UK, 1995)

[9] Laverdant J, Buil S, Hermier J-P and Quélin X 2010 J Nanophotonics 4040505

[10] Introduction to Complex Mediums for Optics and Electromagnetics, Eds Weigelhofer W S and Lakhtakia A (SPIE Press, Bellingham WA, USA, 2003)

[11] Schmelzer J, Brown S A, Wurl A, Hyslop M and Blaikie R J 2002 Phys. Rev. Lett. 88 226802

[12] Tuncer E and Niklasson G A 2008 Optics Commun. 2814374

[13] Smith G B and Granqvist C G Green Nanotechnology: Solutions for Sustainability and Energy in the Built Environment, Chapter 3 ( CRC Press, Boca Raton, Fl, USA, 2010)

[14] Smith G B, Gentle A R and. Maaroof A I J Nanophotonics 20071013507

[15] Smith G B and Earp A E Nanotechnology 201021015203 
[16] Maaroof A I, Cortie M B, and Smith G B, 2005 J. Optics A. Pure and Applied Optics, 7, 1

[17] Earp A A and Smith G B 2010 Appl. Phys. Lett. 96243108

[18] Oldenburg S J, van Blaaderen A and Halas N 1998 Chem. Phys. Lett. 288243

[19] Niklasson G A and Granqvist C G 1985 Appl. Phys. Lett. 46713

[20] McLachlan D S 1987 J Phys. C 20865 